\title{
Generation of Soliton Molecules with Independently Evolving Phase in a Mode-Locked Fiber Laser
}

\author{
Bülend Ortaç, ${ }^{1,+}$ Alexandr Zaviyalov, ${ }^{2}$ Carsten K. Nielsen, ${ }^{1,3}$ Oleg Egorov, ${ }^{2}$ \\ Rumen Iliew, ${ }^{2}$ Jens Limpert, $^{1}{ }^{\text {Falk Lederer, }}{ }^{2}$ and Andreas Tünnermann ${ }^{1,4}$ \\ ${ }^{1}$ Institute of Applied Physics, Friedrich-Schiller-Universität Jena, Max-Wien-Platz 1, 07743 Jena, Germany \\ ${ }^{2}$ Institute of Condensed Matter Theory and Solid State Optics, Friedrich-Schiller-Universität Jena, Max-Wien-Platz 1, \\ 07743 Jena, Germany \\ ${ }^{3}$ University of Aarhus, Department of Physics, DK-8000 Aarhus, Denmark \\ ${ }^{4}$ Fraunhofer Institute for Applied Optics and Precision Engineering, 07745 Jena, Germany \\ ${ }^{+}$Present address, UNAM-Institute of Materials Science and Nanotechnology, Bilkent University, 06800 Ankara, Turkey \\ e-mail: aleksandr.zavyalov@uni-jena.de
}

\begin{abstract}
We report the experimental generation of two-soliton molecules in an ytterbium-doped fiber laser. These molecules exhibit an independently evolving phase and are characterized by a regular spectral modulation pattern with a modulation depth of $80 \%$.

(C) 2010 Optical Society of America

OCIS codes: (140.3510) Lasers, fiber; (190.0190) Nonlinear optics; (190.5530) Pulse propagation and temporal solitons
\end{abstract}

The generation of soliton molecules, also frequently termed bound states, in mode-locked fiber lasers attracted a great deal of interest because of their potential applications in communication lines, optical logic systems, and high resolution optics. Usually such molecules consist of two pulses and are characterized by the peak-to-peak separation and the phase difference between both pulses. Theoretically, a few kinds of stable scalar soliton molecules have been discovered in mode-locked fiber lasers, namely molecules with an invariant phase, or molecules which are slightly vibrating or shaking around a stationary state, molecules with an independently evolving phase and such with a flipping phase. Experimentally, only soliton molecules with an invariant phase [1] and vibrating molecules have been reported to date [2]. This may be attributed to practical difficulties in the measurement of minor changes of molecule parameters or of the complex internal dynamics like periodic or chaotic evolution of the phase difference between the pulses.

In the present Letter we report the first experimental observation of robust two-soliton molecules with an independently evolving phase in a mode-locked fiber laser. The laser operates in the normal dispersion regime and is based on a polarization-maintaining (PM) fiber that guarantees the scalar nature of the solitons. A distinct feature of these molecules is the regularly modulated spectrum with a modulation depth significantly less than $100 \%$. The experimental results are confirmed by numerical modeling backing the genuine observation of this type of soliton molecule.

The experimental configuration of the passively mode-locked wave-breaking-free fiber laser is shown in Fig. 1. All-fiber components are based on the PM single-clad concept. This scalar platform is a very attractive configuration to study soliton molecules dynamics since a single polarization state propagates inside the cavity. A section of $31 \mathrm{~cm}$ highly Yb-doped fiber is spliced between the identical lengths of passive fiber-based components. Passive modelocking is achieved by using a high-modulation depth (30\%) and short relaxation time ( 500 fs) saturable absorber mirrors (SAM). The passive fiber length inside the cavity is $5.6 \mathrm{~m}$. In our experiment, the distance between the gratings is $1.6 \mathrm{~cm}$, which leads to a total round-trip positive group delay dispersion of $+0.03 \mathrm{ps}^{2}$ at $1035 \mathrm{~nm}$. Thus the laser operates in the normal dispersion regime. The output signal is analyzed by a commercial autocorrelator and an optical spectrum analyzer (Ando AQ6315A) requiring a few minutes to record a spectrum. A 50-GHz oscilloscope (Tektronix TDS 8000) and a 25-GHz high-speed photodetector (New Focus 1434) are used to study the time evolution of the laser output.

When the pump power reaches $125 \mathrm{~mW}$, the laser delivers two-pulse molecules. Figure 2(a) shows the autocorrelation trace of such a soliton molecule. The separation distance between the pulses is 18.7 ps what equals the delay time from the central to the side-peaks in the figure. The output pulses show an autocorrelation width of $8.3 \mathrm{ps}$, which corresponds to a pulse duration of 5.35 ps assuming a deconvolution factor of 1.55 . The optical spectrum of the soliton molecule is strongly modulated as a direct consequence of the coherence of the pulses. The modulation period is $0.19 \mathrm{~nm}$ which corresponds to a peak separation of 18.7. It is important to note that the spectrum is regularly modulated with just $80 \%$ of modulation depth caused by the averaging effect over many pulses 
in the experimental measurement. Moreover, especially this unmodulated part of the spectrum allows us to conclude that we observe the soliton molecule with evolving phase. These results differ from previous works where fully modulated spectra were reported which correspond to two-pulse molecules with fixed separation distance and invariant phase relation between pulses. Simultaneously, our results show significant differences to those for the vibrating molecules which exhibit a blurred modulated spectrum [2].

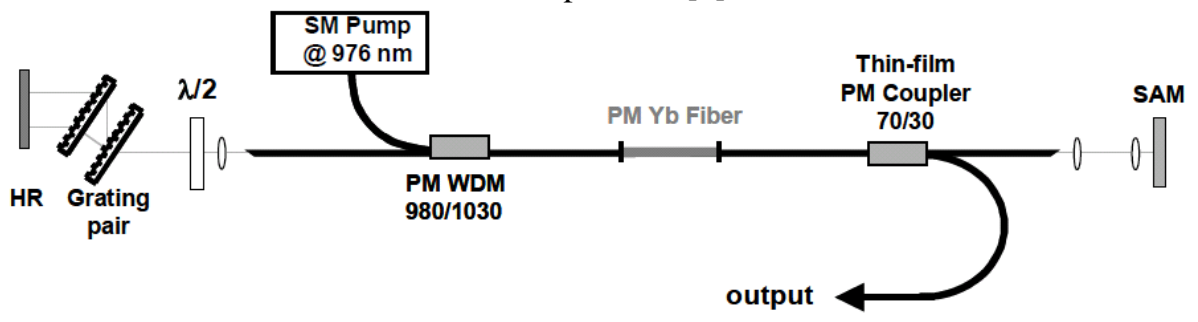

Fig. 1. Experimental setup of the mode-locked fiber laser.

We confirm our claim that the laser works in the independently evolving phase regime by numerical simulations. We use a lumped model where all individual elements are separately described, for details see [3]. The fiber components and the dispersion delay line are described by a modified nonlinear Schrödinger equation which is given by

$$
\frac{\partial U(z, t)}{\partial z}+\frac{i}{2}\left(\beta_{2}+i g(z) T_{1}^{2}\right) \frac{\partial^{2} U(z, t)}{\partial t^{2}}=\frac{g(z) U(z, t)}{2}+i \gamma|U(z, t)|^{2} U(z, t),
$$

where $g(z)$ is saturable gain of the fiber. For the SAM we use the Agrawal/Olsson model:

$$
\begin{aligned}
& \frac{\partial U(z, t)}{\partial z}=-\frac{1}{2} \delta(z, t) U(z, t) \\
& \frac{\partial \delta(z, t)}{\partial t}=\frac{\delta_{0}-\delta(z, t)}{T_{\text {relax }}}-\frac{\delta(z, t)|U(z, t)|^{2}}{E_{\text {sat }}^{S A}}
\end{aligned}
$$

The numerical results are shown in Fig. 2. The spectrum was calculated as average over many periods of the phase oscillations. Excellent agreement between calculated and measured optical spectra can be recognized. Specifically, experiments and simulations yield spectra of equal width, same parabolic shape, and similarly regularly modulated. There is a minor difference in the modulation depth (experiment $\sim 80 \%$ and simulations $\sim 70 \%$ ). The intensity profile of the simulated soliton molecule is almost identical to that being experimentally observed. This is proven by almost identical intensity autocorrelation functions. Using the inherent advantages of modeling properties, which are experimentally not easily accessible, can be identified as the temporal evolution of this molecule, the evolution trajectory on the phase plane and the phase difference between pulses as function of the round-trip number. Unfortunately the difference of the amplitudes cannot be recognized in both the experimental and the simulated autocorrelation functions for its smallness, but it can be clearly seen from the simulated propagation of the soliton molecule in Fig. 2(d). Thus, the numerical results reproduce the experimental ones with high accuracy and confirm that the obtained soliton molecule exhibits an independently evolving phase which causes the partly unmodulated averaged spectrum profile.
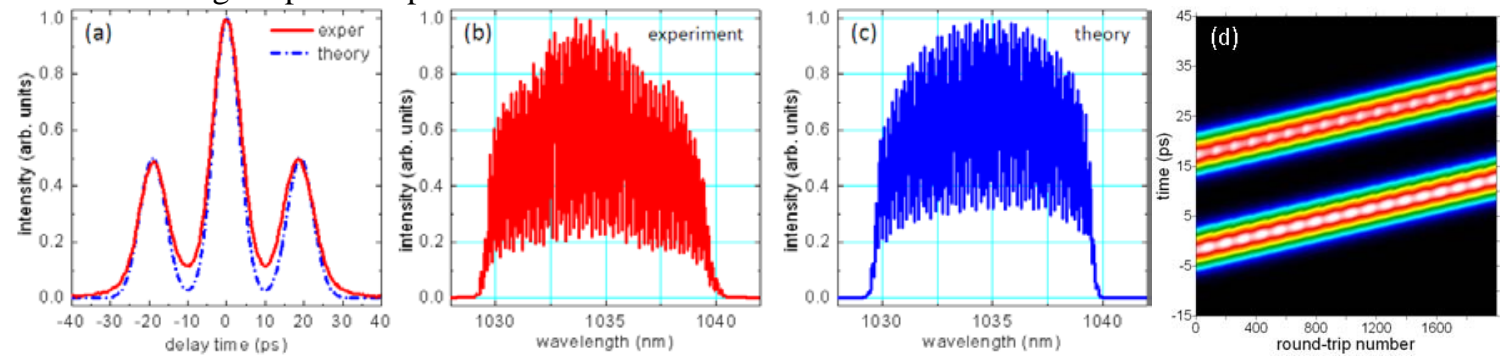

Fig. 2. Experimentally measured and numerically calculated intensity autocorrelation function (a) and optical spectrum (b - experiment, c modeling) of a soliton molecule. Simulated evolution of the molecule (d).

[1] A. Hideur, B. Ortac, T. Chartier, M. Brunel, H. Leblond, F. Sanchez, "Ultra-short bound states generation with a passively mode-locked highpower Yb-doped double-clad fiber laser,” Opt. Comm. 225, 71 (2003).

[2] M. Grapinet, P. Grelu, "Vibrating soliton pairs in a mode-locked laser cavity,” Opt. Lett. 31, 2115 (2006).

[3] A. Zavyalov, R. Iliew, O. Egorov, F. Lederer, "Discrete family of dissipative soliton pairs in mode-locked fiber lasers,” Phys, Rev. A 79, 053841 (2009). 